The new Biolmaging column of BioTechniques will feature short articles devoted to microscopy and digital imaging. The subject matter will address details of the methods used to produce images of cells and tissues at any magnification and resolution, and might include "tricks-of-the-trade", novel methods of specimen preparation, practices of image collection, tips on the digital manipulation and publication of images and historical perspectives.

\section{Scientific Imaging with Digital Cameras}

\author{
Kenneth R. Spring \\ National Institutes of Health, National Heart, Lung \\ and Blood Institute, Bethesda, MD, USA
}

"The picture was taken with a digital camera" is heard more and more often today. This article describes what a digital camera is, how it differs from a video camera and how to select one for your application.

\section{Digital vs. Analog Cameras}

When a charge-coupled device (CCD) camera incorporates an analog-to-digital (A/D) converter on the sensor or in close proximity, we call it a digital camera. Since CCD chips, like all optical sensors, are analog devices that produce a stream of varying voltages, the term digital is used only when those voltages are digitized in the camera and output in a computercompatible format. In a 12-bit digital camera, the analog signal from the CCD is digitized with 12-bit depth by the on-board A/D converter. Whether or not the output can actually be resolved into 4096 ( $2^{12}$ or 12 bits) discrete intensity levels depends on the camera noise. To discriminate between individual intensity levels, each gray level step should be about 2.7 times larger than the camera noise. Otherwise, the difference between steps 2982 and 2983, for example, cannot be resolved with certainty (for additional information, see Chapter 12 in Reference 1). Some so-called 12-bit cameras have so much camera noise that 4096 discrete steps cannot be discriminated. The sources and magnitudes of camera noise will be considered below.

\section{Why Digital?}

If the signal is analog to start with, why digitize it in the camera rather than somewhere downstream? There are two benefits to using an in-camera A/D converter: reduced noise and direct computer-compatible output. In general, the closer the $\mathrm{A} / \mathrm{D}$ is to the sensor, the lower the noise. Low-level analog signals from the CCD are far more readily corrupted by noise than their high-level digital counterparts. In the ideal case, the $\mathrm{A} / \mathrm{D}$ is on the CCD chip, immediately adjacent to the output amplifier of the sensor. The lower the noise, the more gray levels can be identified and, therefore, the more bits that can be meaningfully used for the intensity measurement.

A digital camera has several advantages over its analog counterpart. Digital cameras produce a progressive scan output unlike the interlaced signal generated by video cameras. Digitization of interlaced video signals requires specialized capture boards and frame buffers. The output of progressive scan cameras can be interfaced directly to the computer (e.g., IEEE-1394, RS-422 or SCSI interfaces). In a progressive scan camera, the entire image is first acquired during the exposure time (also denoted as the integration period) and then read out, line by line from the top of the image to the bottom. Modern, high-speed amplifiers and A/D converters permit digital cameras to produce full-frame images at rates that equal or exceed the video framing rate.

The output of a digital camera perfectly suits the format of a computer monitor. Since the signal is already digitized, image storage, manipulation and display are greatly simplified in comparison with similar maneuvers of video signals. The difficulties of dealing with prints, slides and negatives are eliminated in digital photography because many scientific journals now accept digital image files. The result is improved quality both of published images and those shown in presentations. The digitized image can be processed (2), compressed, transmitted via the Internet, pasted into documents or turned into a poster (Figure 1).

\section{CCD Architecture}

Two CCD designs are commonly used in digital cameras: interline transfer and frame transfer. The interline-transfer CCD incorporates charge transfer channels beside each photodiode so that the accumulated charge can be efficiently and rapidly shifted over to them (Figure 2). Interline-transfer sensors can also be electronically shuttered "off" by dumping the stored charge instead of shifting it into the transfer channels. The frame-transfer CCD uses a two-part sensor in which the top half is covered by a light-tight mask and is used as a storage region. Light is allowed to fall on the uncovered portion, and the accumulated charge is then rapidly shifted into the masked storage region. While the signal is being integrated on the lightsensitive portion of the sensor, the stored charge is read out.

Two types of color digital cameras are used for scientific applications - a single CCD camera with a wavelength selection filter or a three-sensor camera. Both use filters to produce red, green and blue versions of the field-of-view. The singlesensor camera uses a filter wheel or liquid-crystal tunable filter to acquire the red, green and blue images in sequence. The three-sensor camera has a beam-splitting prism and trim filters that enable each sensor to image the appropriate color and to acquire all three images simultaneously. Invariably, color cameras are less sensitive than their monochrome counterparts because of the additional beam-splitting and wavelength selection components. In some applications, particularly immunofluorescence, the loss of sensitivity is offset by the ability to capture multiple wavelengths simultaneously or in rapid 


\section{Bielmaging}

succession. In addition, some color cameras achieve a higher resolution by diagonally offsetting the red, green and blue sensors, each by one-third of a pixel, thereby tripling the number of samples obtained.

Although CCD camera manufacturers and users routinely refer to each photodiode as a pixel (picture element), there is no requisite correspondence between the number and position of the pixels in the sensor and those in the computer monitor or printer. However, the display or printer resolution should always be at least as high as that of the sensor.

\section{Quantum Efficiency}

Quantum efficiency $(\mathrm{QE})$ refers to the percentage of incident photons that are detected. (For reference purposes, the QE of our photopic vision is about 3\%.) Silicon photodiodes, the basic building blocks of the CCD, have a high QE (80\%) across a broad range of the visible spectrum and into the near infrared. The spectral sensitivity of a CCD is lower than that of a simple silicon photodiode because the CCD has charge transfer channels on its surface that reduce peak QE to about $40 \%$. Recently, the transparency of the channels of some scientific-grade CCDs has been increased and the QE in the blue-green range improved to nearly $70 \%$. The losses from

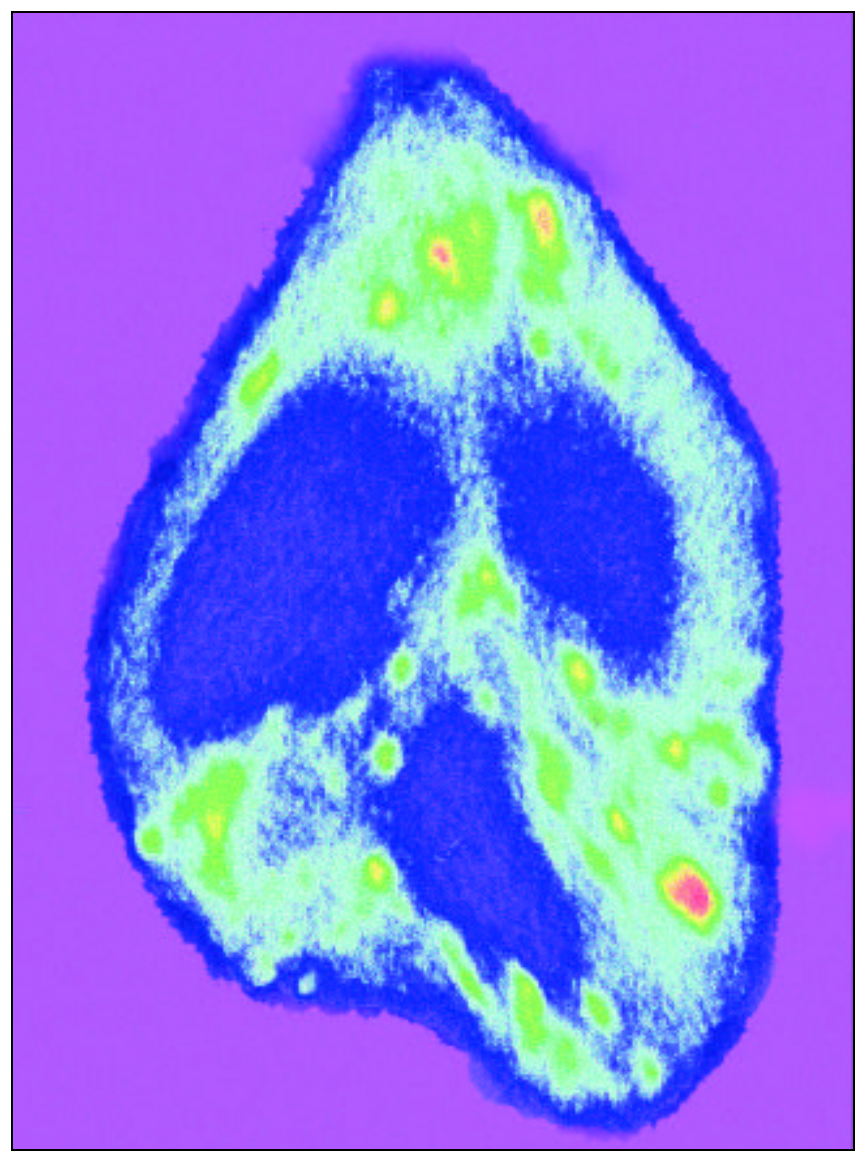

Figure 1. A digital image of a neutrophil stained with antibodies to CD55. The original image, courtesy Dr. Elaine Sloand, has been distorted and pseudocolored using image processing software. the surface channels are completely eliminated in the back-illuminated CCD. In this design, light falls onto the back of the $\mathrm{CCD}$ in a region that has been thinned by etching until it is transparent. A quantum efficiency as high as $90 \%$ can be realized. However, back-thinning results in a delicate, relatively expensive sensor that, to date, has only been employed in scientific-grade, slow-scan CCD cameras.

\section{Noise in CCD Cameras}

There are two major sources of noise in CCD camerasdark noise and read-out noise. Although great improvements have been made over the past few years in the reduction of CCD dark noise at room temperature, cooling the chip further reduces the noise tenfold per $20^{\circ} \mathrm{C}$ decrease. Dark noise is most evident as "hot" pixels (white dots) in images obtained with room temperature CCD cameras after integration periods of 4 or $5 \mathrm{~s}$. Cooling to $0^{\circ} \mathrm{C}$ is usually sufficient for integration periods up to $30 \mathrm{~s}$. Experiments requiring very long exposures (e.g., chemiluminescence) need even lower sensor temperatures. Digital cameras are available in cooled or uncooled versions.

Read-out noise is generated in the amplifier on the CCD chip that converts the stored charge of each photodiode (i.e., pixel) into an analog voltage to be quantified by A/D conversion. Read-out noise may be viewed as a "toll" that must be paid for reading the stored charge. The size of this toll has decreased steadily in the past few years to 5-10 electrons/pixel because of improvements in CCD design, clocking and sampling methods (1). Read-out noise increases in proportion to read-out speed. The cost of going faster is more noise and, hence, more uncertainty in the voltage determination and lower number of bits of resolution. This is why slow-scan cameras generally exhibit lower read-out noise than faster

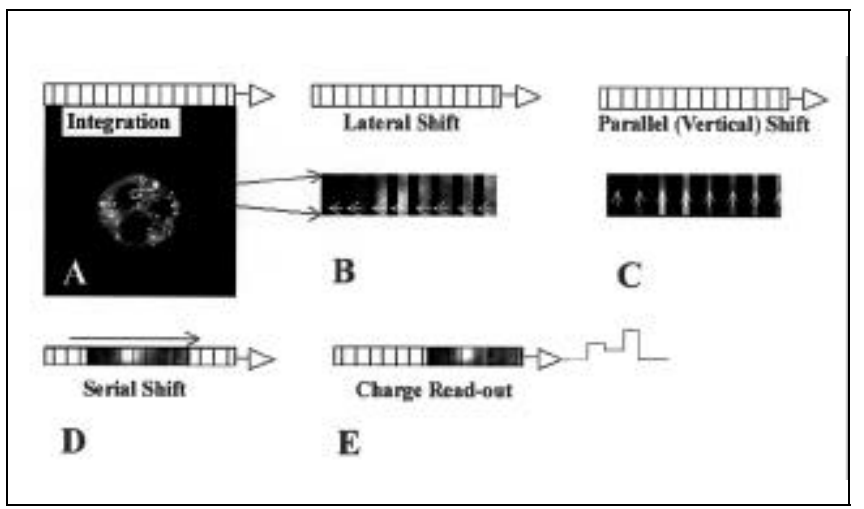

Figure 2. The sequence of operations in an interline-transfer CCD sensor. (A) The original image used in Figure 1 is projected onto the CCD surface during the integration period. (B) A highly magnified view of the region in A outlined by the box, showing the lateral shift of charge from each photodiode to the adjacent charge transfer channel (dark bars). (C) The stored charge in the channels is shifted vertically toward the top of the sensor. The photodiodes are now depicted as the dark regions, while the transfer channels contain the image information. (D) The transfer of charge in the serial register toward the read-out amplifier. (E) Some of the charge has been read out and is depicted as variations in the output voltage of the amplifier. The remaining pixels in the serial register await the shifts necessary to move them to the output amplifier. 
output detectors and have a higher number of useful bits. Digital cameras range from those with 8-12-bit depth at 30 frames/s output to 16-bit depth at 1-2 frames/s.

One solution to the speed/read-out noise problem is the use of multiple output amplifiers (taps) on a large CCD. Instead of reading the stored charge from the entire CCD through one output amplifier, the sensor is divided into four or eight sections each of which has its own amplifier. The image is read out in parts and then stitched together in software at rates of several frames/s. The required speed and associated noise of each amplifier are reduced accordingly.

\section{Signal-to-Noise Ratio}

Since photons randomly arrive at the sensor surface, their numbers fluctuate with a noise as described by Poisson statistics that is equal to the square root of the number of detected photons $(1,3)$. Of course, camera noise adds to this photon statistical noise and further reduces the $\mathrm{S} / \mathrm{N}$. The highest $\mathrm{S} / \mathrm{N}$ that can be achieved by a digital camera is the square root of the maximum accumulated charge (the full-well capacity). A simple estimate of the $\mathrm{S} / \mathrm{N}$ of any homogeneous region in an image is the average intensity of the region of interest divided by the standard deviation of the intensity of that region.

\section{How Many Pixels in a Digital Camera Are Enough?}

The resolution of a CCD is a function of the number of photodiodes and their size relative to the projected image. CCD arrays of $1000 \times 1000$ photodiodes are now commonplace in digital cameras. The trend in consumer and scientific-grade CCD manufacture is for the sensor size to decrease, with some CCD photodiodes as small as $4 \times 4 \mu \mathrm{m}$. From sampling theory, adequate resolution of an object can only be achieved if at least two samples are made for each resolvable unit. (Many users prefer three samples per resolvable unit to ensure sufficient sampling.) In an epifluorescence microscope, the Abbé diffraction limit of a 1.4-NA lens at $550 \mathrm{~nm}$ is $0.22 \mu \mathrm{m}$. For a $100 \times$ objective lens, the projected size of a diffraction-limited spot on the face of the CCD is $22 \mu \mathrm{m}$. A photodiode size of $11 \times 11 \mu \mathrm{m}$ would just allow the optical and electronic resolution to be matched, with a $7 \times 7 \mu \mathrm{m}$ photodiode preferred. With a $100 \times$ objective and no additional magnification, a $1000 \times 1000 \mathrm{CCD}$ with $7 \times 7 \mu \mathrm{m}$ photodiodes would capture a field-of-view of $70 \times 70 \mu \mathrm{m}$ in the object plane. When the size of the image projected onto the CCD is appropriately adjusted for proper sampling, a larger number of photodiodes in the CCD increases the field-ofview, not the resolution.

The resolution requirements of various output devices may require oversampling at the sensor so that the final product (e.g., slide, print or poster) has adequate resolution at the final size.

\section{Dynamic Range}

Intrascene dynamic range denotes the useful range of intensities that can be simultaneously detected in the same field-of-view. Interscene dynamic range is the range of inten- sities that can be accommodated when detector gain, integration time, lens aperture or other variables are adjusted for differing fields of view. Although small sensors in a CCD are desirable from a resolution viewpoint, they limit the dynamic range of the device. The full-well capacity of a CCD is about 1000 times the cross-sectional area of each photodiode. Thus, a CCD with $7 \times 7 \mu \mathrm{m}$ pixels should have a full-well capacity of 49000 electrons or holes. (A hole is the region of the silicon from which the electron came and constitutes an equally valid and usable measure of detected photons. The term electrons is used, although most CCDs read out the number of holes generated rather than electrons.) Since CCDs do not have inherent gain, one electron-hole pair is accumulated for each detected photon. The intrascene dynamic range of a CCD is typically defined as the full-well capacity divided by the camera noise. The camera noise is calculated as the square root of the sum of the squares of the dark and read-out noise. Thus, the dynamic range of a 49000 electron full-well capacity CCD with 10 electrons of read-out noise and negligible dark noise is about 4900 , corresponding to 12 bits. However, digitization of the output from such a camera at 12-bit depth means that the 49000 electrons are divided into $4096 \mathrm{~A} / \mathrm{D}$ units, each containing 12 electrons (49000/4096). Since the noise is 10 electrons, each gray level step is only 1.2 times the noise and cannot be discriminated. Digitization at 10 bits would result in each A/D units being 49 electrons, about five times the noise level, and each of the 1024 gray levels could then be discriminated.

\section{Control of Speed, Effective Pixel Size and Field-of-View}

Slow-scan digital cameras allow for control over the readout rate, the effective size of the pixel that constitutes a sensor and the field-of-view. Scientific-grade CCD cameras usually offer two or more read-out rates so that speed can be traded off against noise. The effective size of a pixel in many slowscan digital cameras can be increased by binning, a process in which the charge from a cluster of adjacent photodiodes is pooled and treated as if it came from a larger detector. Binning is useful when light levels are very low and few photons are detected because it enables the investigator to trade spatial resolution for sensitivity. In addition, most slow-scan CCD cameras allow region-of-interest read-out in which a selected portion of the image can be displayed and the remainder of the accumulated charge discarded. The framing rate generally increases in proportion to the reduction in the field-of-view. For example, a CCD with a sensor size of $1000 \times 1000$ and an output rate of 10 frames/s can produce 100 frames/s if the read-out region is reduced to $100 \times 100$ diodes. By trading off field-of-view and framing rate, an investigator can adjust to a far wider range of experimental circumstances than would be possible with a fixed framing-rate camera.

\section{Intensified Digital Cameras}

Several manufacturers now offer digital cameras equipped with an image intensifier for very low light-level imaging. These have a photocathode in close proximity to a microchannel plate electron multiplier and a phosphorescent 


\section{Useful Web Sites}

www.ShortCourses.com

www.theimagingsource.com

www.videomicroscopy.com

output screen. The photocathode in the latest generation of these devices has a high quantum efficiency (up to 50\%) in the blue-green end of the spectrum. Intensifier gain is adjustable over a broad range with a typical maximum of about 80000 . Thermal noise from the photocathode as well as electron multiplication noise from the microchannel plate reduce the $\mathrm{S} / \mathrm{N}$ in an intensified CCD camera to below that of a slowscan CCD. Resolution of an intensified CCD depends on both the intensifier and the CCD but is usually limited by the intensifier microchannel plate geometry to about $75 \%$ of that of the CCD alone.

Intensified digital cameras have a reduced intrascene dynamic range compared with slow-scan cameras, and most are limited to 10-bit resolution. Intensifier gain may be rapidly and reproducibly changed to accommodate variations in scene brightness, thereby increasing the interscene dynamic range. Indeed, since image intensifiers can be gated, that is, turned off or on in a few nanoseconds, relatively bright objects can be visualized by a reduction in the "on" time. Gated, intensified digital cameras are required for most time-resolved fluorescence microscopy applications because the detector gain must be modulated at high frequency in synchrony with the light source. Because of the low light fluxes required in living cells, intensified CCD cameras are frequently employed to study dynamic events and for ratio imaging (3).

\section{Choosing the Appropriate Camera for Your Application}

No single detector will meet all requirements in fluorescence microscopy, so the investigator must compromise. Exposure time is often the critical parameter. When time is available for image integration, a slow-scan CCD camera will outperform an intensified camera in all areas, in large part because of its higher quantum efficiency and lower noise. Cooling always improves digital camera performance, although the difference may not be noticeable when the integration time is a few seconds or less and the digitization depth is 8-12 bits. For applications involving digital deconvolution, the detector of choice is a cooled, scientific-grade, slow-scan camera capable of producing a high-resolution, 14-16-bit image. Photodiode size matters; some CCDs have such small pixels that the integration period may have to be limited to avoid saturation of the charge storage wells, with the result that the dynamic range and peak $\mathrm{S} / \mathrm{N}$ may be compromised. If the event under investigation is rapid but can be precisely triggered, then a slow-scan CCD operating in a burst or high-speed mode may be suitable. However, when the event is not readily predictable and the specimen must be monitored continuously at low-incident light flux, the intensified CCD is the detector of choice. For this reason, single-molecule fluorescence imaging uses an intensified digital camera.

When color images are needed of routine histological specimens, the three-CCD camera is preferable to an inexpensive single-sensor camera with an integral color mask. Highresolution, single-sensor CCD cameras equipped with a removable, red-green-blue, liquid-crystal filter have proven very useful for both brightfield and fluorescence microscopy.

\section{Future Prospects}

Recent improvements in the performance of CMOS (complimentary metal oxide semiconductor) cameras herald a potentially important future role for these devices in fluorescence microscopy. CMOS cameras have an amplifier and digitizer associated with each photodiode in an integrated onchip format. The result is a low-cost, compact, versatile detector combining the virtues of silicon detection without the problems of charge transfer. CMOS sensors allow gain manipulation of individual photodiodes, region-of-interest readout, high-speed sampling, electronic shuttering and exposure control. They have extraordinary dynamic range and an ideal format for the computer interface. Until recently, they suffered from high fixed-pattern noise associated with switching and sampling artifacts, but these problems are now being solved rapidly. It is likely that they will replace the CCD in digital cameras for a number of scientific applications in the near future.

\section{REFERENCES}

1.Inoué, S. and K.R. Spring. 1997. Video Microscopy, The Fundamentals. Plenum Press, New York.

2.Russ, J.C. 1995. The Image Processing Handbook., 2nd ed. CRC Press, Boca Raton, FL.

3.Sluder, G. and D.E. Wolf. 1998.Video Microscopy. Academic Press, San Diego, CA.

Address correspondence to Dr. Kenneth R. Spring, NIH, Building 10, Room 6N260, 10 Center Drive, Bethesda, MD 20892-1603, USA. e-mail: springk@fido.nhlbi.nih.gov

Suggestions for contributions to the Biolmaging feature are welcomed by its editor, Dr. Steve Paddock (paddock@facstaff.wisc.edu) 